\title{
Mapa energético-solar de Concepción: cartografía urbana del consumo energético y captación solar en edificaciones residenciales de Concepción, Chile ${ }^{1}$
}

\author{
Rodrigo García Alvarado², Paulina Wegertseder ${ }^{3}$, Sergio Baeriswyl ${ }^{4}$ \\ y Maureen Trebilcock ${ }^{5}$
}

\begin{abstract}
RESUMEN
Con el fin de promover el uso de la energía solar en las ciudades, se presenta una metodología de catastro urbano del consumo energético y captación solar, aplicada para distintas tipologías residenciales, categorizadas por manzanas, en Concepción, Chile. La categorización se realiza mediante encuestas, registros socioeconómicos, fotos aéreas, tipologías de edificación y simulaciones, que se implementan en un Sistema de Información Geográfico (SIG). Se identifican rangos y zonas, que entregan como primer resultado, una distribución diferenciada de consumos y potencialidades solares, que constituyen la base para implementar una matriz de energía renovable. El estudio muestra que en Concepción los sectores de alta densidad presentan menor potencial de captación solar, y los sectores con baja densidad muestran un sobrepotencial, que permitiría alcanzar un equilibrio en la matriz energética solar de la ciudad, mediante la transferencia entre los sectores.
\end{abstract}

Palabras clave: Energía solar, consumo energético, mapas urbanos, tipologías residenciales, Chile.

\begin{abstract}
With the aim of promoting solar energy use in cities, this paper presents an urban assessment of energy consumption and incident solar radiation, applied to different residential typologies, categorized by block in Concepción, Chile. The categorization was done through surveys, socioeconomic records, aerial photographs, building typologies and simulations, which were implemented in a Geographic Information System (GIS). The identified ranges and zones give, as a first result, the differential distribution of energy consumption and solar potential, which constitute the basis for implementing an array of renewable energy. This work shows that high-density sectors have less solar potential in Concepción, whereas low-density sectors show a surplus, which could allow achievement of a balance in the solar energy matrix by energy transfer between sectors of the city.
\end{abstract}

Key words: solar energy, energy consumption, urban maps, residential typologies, Chile.

\footnotetext{
Este artículo agradece a AKA-Conicyt ERNC 007 "Assessment of Solar Energy Potential in Urban Areas", a los profesores Dr. Adelqui Fissore de la Universidad de Concepción y Dr. Peter Lund de la Universidad Aalto, Helsinki; y finalmente a los asistentes Laline Cenci, Cristián Cuevas, Andrés Rodríguez, Patricio Loo, Laboratorio de Estudios Urbanos de la Universidad del Bío - Bío. Artículo recibido el 23 de diciembre de 2013, aceptado el 20 de agosto de 2014 y corregido el 30 de septiembre de 2014
}

2 Universidad del Bío-Bío (Chile). E-mail: rgarcia@ubiobio.cl

3 Universidad del Bío-Bío (Chile). E-mail: pwegertseder@ubiobio.cl

4 Universidad del Bío-Bío (Chile). E-mail: sbaerisw@ubiobio.cl

5 Universidad del Bío-Bío (Chile). E-mail:mtrebilc@ubiobio.cl 
A nivel mundial, las edificaciones son responsables del $60 \%$ de las emisiones de $\mathrm{CO}_{2}$ a la atmósfera (IEA, 2009), siendo gran parte de esta contaminación consecuencia de la energía requerida para su funcionamiento, especialmente en términos de climatización. Además, el crecimiento socioeconómico y el constante aumento de la urbanización (especialmente en Latinoamérica), evidencia que las ciudades se están convirtiendo en los principales centros de consumo energético e impacto ambiental (Balbo et al., 2003). Su demanda es satisfecha principalmente por combustibles no renovables provenientes de fuentes alejadas, lo que provoca un costo cada vez mayor de la energía para los usuarios.

Por esta razón se advierte la necesidad de lograr procedimientos de autogeneración energética en la ciudad y sus edificaciones, preferentemente de fuentes sustentables disponibles en el propio lugar, como la energía solar (Meador, 1981). Sin embargo el consumo de las edificaciones y su posibilidad de captar la radiación solar, poseen una distribución espacial y temporal diferente (Lund, 2010). La demanda energética aumenta en las noches y periodos invernales, mientras la captación solar es mayor durante el día y en verano. Del mismo modo, las edificaciones densas concentran el gasto y la captación solar se facilita en lugares despejados. Debido a esto es crucial determinar la estructura urbana de los requerimientos energéticos y de las posibilidades de recolección solar, para definir el tipo de instalaciones más adecuadas y complementar la provisión actual de combustibles. Buscando orientar la inversión privada y la gestión pública de fuentes renovables locales para alcanzar ciudades más sustentables.

Actualmente Chile presenta una crisis múltiple en el sector energético, debido a altos niveles de contaminación, elevados precios de energía, una matriz de generación escasamente diversificada y concentrada en fuentes fósiles mayoritariamente de origen extranjero, inseguridad en el abastecimiento por restricciones ambientales, inequidad en la distribución eléctrica y retraso de políticas energéticas reguladoras y promotoras de nuevas fuentes (CCTP, 2011). El país ha entrado en una situación vulnerable, demandando acciones que incentiven la eficiencia energética y el uso de energías renovables, sin sacrificar el confort o la actividad económica (Castillo \& Maldonado, 2004). Algunas acciones públicas en Chile están motivando la implementación de fuentes renovables, como la ley 20.365 promulgada en 2009, que establece franquicia tributaria a sistemas solares térmicos para Agua Caliente Sanitaria (ACS). En 2012 se dicta la ley 20.571 que viabiliza el "Net-Metering" o venta de energía eléctrica generada a escala doméstica para la red pública. Por este motivo han surgido distintos proveedores, pero aún su aplicación es muy incipiente porque entre otras cosas, se carece de información precisa sobre sus posibilidades de uso y las condiciones del entorno urbano.

La captación de energía solar para consumo directo en el lugar (on-site), permite prescindir de redes de transporte y distribución, evita la construcción, mantención y contaminación espacial de grandes sistemas (Widén, 2010). El presente estudio pretende entonces promover la aplicación de energías renovables en las ciudades, específicamente en el uso residencial, determinando la zonificación de sus requerimientos y posibilidades de captación solar. Se identifica la distribución del gasto habitacional (eléctrico, agua caliente sanitaria y calefacción) por sectores, y las capacidades de radiación recibidas en las propias edificaciones, posible de ser aprovechadas. Estableciendo la zonificación de estas variables según la estructura urbana y tipologías residenciales. Como se carece de información de servicios centrales o levantamientos tridimensionales (que son utilizados en países desarrollados para realizar este tipo de catastros), en esta investigación se implementa una categorización territorial por manzanas de acuerdo a bases cartográficas, encuestas de gasto domiciliario, niveles socioeconómicos, fotos aéreas, modelos habitacionales y simulación dinámica, implementada en un Sistema de Información Geográfico (SIG). En este artículo se explica el proceso aplicado en la ciudad de Concepción, Chile y los principales resultados obtenidos.

\section{Catastros energéticos y captación solar urbana}

La distribución de los requerimientos energéticos de las ciudades se puede determinar a 
partir de la información de los reguladores o proveedores de servicios, o de las propias edificaciones, por sus registros de consumo o por simulaciones dinámicas para comprender sus demandas detalladas. Estos catastros se enfrentan a dilemas del nivel de detalle y de la relación de variada información, incluyendo la composición poblacional y su proyección en el tiempo (Bunje et al., 2012).

Recientemente se han divulgado varios estudios y catastros energéticos de ciudades para promover un desarrollo urbano más sustentable. Por ejemplo de las ciudades norteamericanas de Los Angeles (LA) (CCSC, 2013) y Nueva York (NY) (SEL, 2013), como también varias de la región de Ontario en Canadá (CUI, 2013). Los catastros de LA y NY, que están disponibles en mapas interactivos por internet, detallan fundamentalmente los consumos eléctricos por manzana (o agrupaciones de manzanas). Aunque no abarcan la totalidad de las áreas metropolitanas y servicios energéticos, despliegan información asociada de uso de suelo, cantidad de población, antigüedad media de las construcciones y algunos especifican consumos energéticos mensuales, permitiendo observar la variación entre un mes y otro. El mapa de LA presenta una escala de consumo desde $30 \mathrm{a} 3.000 \mathrm{kWh} / \mathrm{m}^{2}$ año promedio, incluyendo sectores residenciales, comerciales y otros.. El mapa de NY, sustentado en datos comunales de consumo eléctrico y encuestas, revela variaciones de 50 a $5.000 \mathrm{kWh}$ anuales por $\mathrm{m}^{2}$, donde Manhattan supera los $1.000 \mathrm{kWh} / \mathrm{m}^{2}$, y las zonas residenciales periféricas (Bronx, Brooklyn y Queens) desperdigándose bajo los $300 \mathrm{kWh} / \mathrm{m}^{2}$ año. El estudio general de este catastro aporta además el análisis de distintas fuentes y proyecciones (Howard et al., 2012). De las ciudades canadienses revisadas, Guelph presenta consumos promedio de 30 a 280 $\mathrm{kWh} / \mathrm{m}^{2}$ al año incluyendo distintas fuentes. Aunque no está disponible en cartografía interactiva, el mapa revela áreas industriales laterales, zonas residenciales medias y centros históricos bajos e irregulares (Gilmour et al., 2010). También revisando distintos combustibles y estimaciones en el tiempo.

Por otro lado, han surgido variados registros territoriales de captación solar para incentivar la utilización de recursos renovables locales, dirigidos a residentes, instaladores de sistemas, financiadores y gobiernos, entre otros. Basados en información climática y topográfica satelital (Pérez et al., 2002; Janjai et al.; 2005), implementada en sistemas de información geográfica (SIG) para identificar las variación geográficas de incidencia solar y estimar magnitudes globales de contribución a las demandas. Por ejemplo, un estudio completo del país de Omán (Gastli \& Charabi, 2010), registra magnitudes medias de $M W h / m^{2}$ año en terrenos planos sugiriendo que la radiación recibida puede aportar más de 680 veces los requerimientos nacionales de energía. Sin embargo no detallan la situación de las zonas urbanas, que presentan diversas condiciones locales. La revisión de áreas construidas se concentra usualmente en la capacidad de recolección solar de las cubiertas de edificios, estimando magnitudes generales por sectores (Izquierdo, 2008), aunque sin considerar fachadas o patios, como tampoco la estructura urbana. Una revisión más detallada del potencial solar de las edificaciones requiere la información volumétrica de las construcciones y el cálculo de radiación recibida por cada frente o lugares anexos, que luego se puede graficar e implementar en sistemas catastrales (UPM \& POLIS, 2010). En un reciente proyecto de investigación desarrollado en Europa, se evaluó el potencial solar en tres ciudades: Lisboa, París y Vitoria-Gateiz. En la primera se estudiaron 60.000 edificios, determinando que $28 \%$ de todos los techos son ideales para la instalación de tecnologías de energía solar con radiación anual de más de $1.600 \mathrm{kWh} / \mathrm{m}^{2}$. En tanto, para París se estudiaron 80.000 edificios de techo planos, concluyendo que si se instalan $200.000 \mathrm{~m}^{2}$ de paneles fotovoltaicos, se alcanzaría 0 GWh de producción eléctrica anual, es decir solo un $0,2 \%$ del consumo estimado para el año 2014 (10.200 GWh). Para la ciudad de Vitoria-Gateiz, el estudio fue más preciso, ya que se consideraron un espectro más amplio de escalas analizadas, desde una visión general de toda la ciudad hasta los edificios individuales. Se calificaron las techumbres según porcentajes de pérdida de la radiación recibida, sugiriendo implicancias en la planificación urbana (Grauthoff et al., 2012). La metodología de análisis de cada ciudad, varió según los datos recopilados y la complejidad de escala, debido al tamaño de 
cada una de ellas. Algunos servicios (como Díaz, 2013), permiten realizar levantamientos digitales tridimensionales de grandes áreas construidas y graficar porcentajes de incidencia solar, para realizar estimaciones de potencial en estudios o mapas interactivos.

Actualmente es posible encontrar diversos mapas urbanos de captación solar en línea, los cuales entregan un catastro de cubiertas de edificaciones, y cálculos adicionales de cuanta energía puede generar instalando sistemas fotovoltaicos o solares térmicos en sus edificaciones, los costos y retornos de inversión. En general abordan ciudades del hemisferio norte, con mayor concentración en Estados Unidos y Alemania. Mapas de la ciudades Emden (ES, 2013), Gelsenkirchen (DS, 2013) y Anaheim (ASM, 2013) muestran resultados de forma general, es decir identifican posibles ahorros e indican idoneidades solares según colores especificados en las techumbres. Otros mapas, como el de la ciudad de Bremen (SB, 2013), Berkeley (CB, 2013), Los Angeles (LAC, 2013) y Cambridge (Reinhart et al., 2013) entregan mayor cantidad de información al usuario o a la empresa que intervendrá, además de la superficie de la cubierta según la idoneidad, muestran la superficie ideal del módulo fotovoltaico, qué rendimiento pudiese este tener, la cantidad de $\mathrm{CO}_{2}$ disminuida y el ahorro energético ( $\mathrm{kWh} / \mathrm{año}$ ). Exponiendo catastros homogéneos de las áreas urbanas, en que destacan los edificios de mayor magnitud continua de techumbre. Estos mapas no consideran espacios abiertos o caras verticales, como tampoco las posibilidades de desarrollarlo en otras localidades o su relación con la estructura urbana.

Los estudios de captación de radiación a solar en base a modelos volumétricos generados por rastreo aéreo (vuelos LIDAR), son onerosos y requieren grandes volúmenes de procesamiento computacional (Lukac \& Zalik, 2013) y de calibración local (Jakubiec \& Reinhart, 2013). Estas condiciones son escasamente existentes en países en desarrollo del hemisferio sur.

Por esta razón planteamos un catastro por manzanas basado en informaciones particulares, que por un lado represente rangos de consumos urbanos según encuestas de población, y por otro estime la captación solar con el cálculo de tipologías constructivas, para determinar diferenciaciones generales y potencial complementario. Asegurando la integridad del modelo mediante la zonificación territorial, para así tener una aproximación tipológica a los requerimientos y potencialidades energéticas que permitan orientar la provisión solar urbana y promover una conciencia ambiental.

Cuando el objetivo es emplear sistemas de energía distribuida en las ciudades, el espacio útil para instalar los equipos puede poner límites a la cantidad de energía que está disponible con respecto a la demanda (Aberg et al., 2012). Por ejemplo, los centros de las ciudades suelen tener edificios altos, los cuales presentan menor capacidad de recolección solar, debido a la poca superficie expuesta (especialmente de techumbre) y sombreamiento entre los volúmenes, en comparación con casas unifamiliares. La morfología constructiva y el relieve inciden directamente en la captación de energía que se puede generar para la demanda energética. Además, el abastecimiento debe equilibrarse o almacenarse durante los periodos diarios o anuales para satisfacer necesidades efectivas de energía (Lund, 2012).

Por otro lado, el desarrollo de la energía solar depende de políticas locales, transferencia de tecnología, costos de los sistemas recolectores y el recurso solar del lugar. Es necesario integrar todos estos factores para planificar la explotación de la energía solar como fuente de producción, como también se deben identificar tres tipos de potenciales: físicos (energía recibida desde el Sol en diferentes periodos temporales); geográficos (características formales de las superficies y conos de sombra); y técnicos (que tipos de equipos usar), con la ayuda de un sistema de información territorial para el análisis espacial, para luego evaluar la vialidad económica (Sun et al., 2013).

\section{Situación de Concepción}

La ciudad de Concepción está ubicada en la zona centro sur de Chile, a $36^{\circ} 46^{\prime} 22^{\prime \prime} \mathrm{S}$ de latitud y $73^{\circ} 03^{\prime} 47^{\prime \prime} \mathrm{O}$ de longitud (Figura $N^{\circ} 1$ ). Con una elevación promedio cercana a los $12 \mathrm{msnm}$, se encuentra a los pies de la cordillera de la Costa y en la ribera norte del 
río Biobío, en planicies litorales del océano Pacífico. Esta condición arroja una geomorfología irregular con colinas, quebradas y depresiones. Su clima es templado marítimo con influencia mediterránea y cuatro estaciones claramente marcadas, presenta en prome- dio $66,5 \%$ de humedad relativa. La temperatura media anual es de $9,1{ }^{\circ} \mathrm{C}$ y la máxima es de $20^{\circ} \mathrm{C}$. El promedio de temperatura en verano es $17^{\circ} \mathrm{C}$, mientras que en invierno la media es $8^{\circ} \mathrm{C}$ (Dirección Meteorológica de Chile, 2013).

Figura $\mathrm{N}^{\circ} 1$

Izquierda, localización de la Región del Biobío en Chile. Derecha, ubicación de Concepción en la Región del Biobío

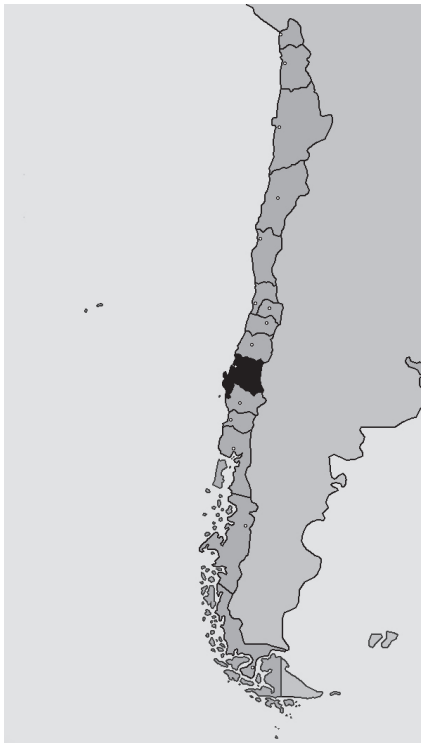

Fuente: Elaboración Propia.

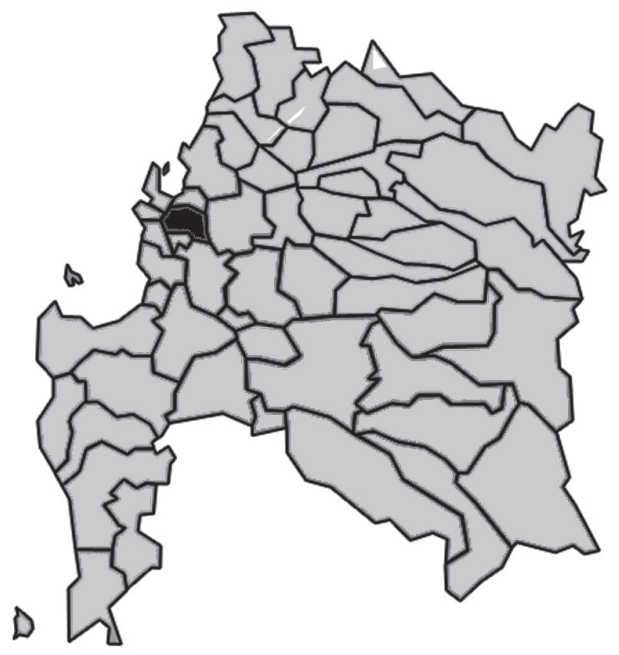

El Área Metropolitana de Concepción es la segunda concentración urbana del país con una población cercana al millón de habitantes, distribuidos en 11 comunas con una extensión urbana de $14.000 \mathrm{ha}^{6}$. La comuna de Concepción es el centro histórico, político y de servicios de este sistema. Para el Censo del año 2002 la Comuna de Concepción contaba con 216.021 habitantes (INE, 2002) con una superficie urbanizada de 2.102 ha, con una densidad habitacional de 103 hab/ha. El área residencial construida representa 76,4\% del total de la superficie comunal (Salinas y Pérez, 2011). Según las proyecciones nacionales del Instituto Nacional de Estadísticas

\footnotetext{
6 Primer Informe Calidad de Vida Urbana Gran Concepción 2011-2012.
}

(INE-CEPAL, 2005), para el 2050 la comuna podría alcanzar más de 250.000 hab. y el área metropolitana sobre los 1.500.000 hab., con variaciones de distribución demográfica.

El Área Metropolitana de Concepción ha crecido de forma similar a la mayoría de las ciudades latinoamericanas, con una concentración de servicios, expansión horizontal en el entorno geográfico, presencia ocasional de informalidad, segregación residencial y diversidad de instalaciones industriales y equipamientos (Borja, 2011).

La irradiación solar mensual obtenida en un plano horizontal en Concepción, corresponde al perfil mensual tipo de una ciudad media en el hemisferio sur, donde se nota una clara disminución durante los 
meses de invierno (Figura $\mathrm{N}^{\circ} 2$ ), obtenido un total anual de $1517 \mathrm{kWh} / \mathrm{m}^{2}$. El mismo perfil se puede obtener de las temperaturas ambientales al interior de un edificio, donde estas disminuyen en comparación al periodo estival. En invierno el flujo de pérdida de energía aumenta y por ende lo hace también el consumo energético para calefaccionar. La demanda energética en una vivienda tipo en Concepción, muestra que al menos $70 \%$ de la energía es para calefaccionar (independiente del combustible que se utilice para poder satisfacerla) de un total de $13,500 \mathrm{kWh} / a n ̃ o$ (Fissore, 2009).

Los valores medios mensuales de la radiación solar en una superficie vertical $\left(34.41^{\circ}\right.$ orientación noreste, respecto al norte geográfico), muestran que el mayor índice de energía solar medio aparece al final del verano (marzo), con un máximo de $4,1 \mathrm{kWh} / \mathrm{m}^{2}$. Luego, el mínimo es alcanzado en el otoño (mayo), con un valor de $1,8 \mathrm{kWh} / \mathrm{m}^{2}$ (Figura $N^{\circ}$ 3) (Fissore \& Fonseca, 2007). Resulta

Figura $\mathrm{N}^{\circ} 2$

Radiación promedio mensual en plano horizontal para Concepción

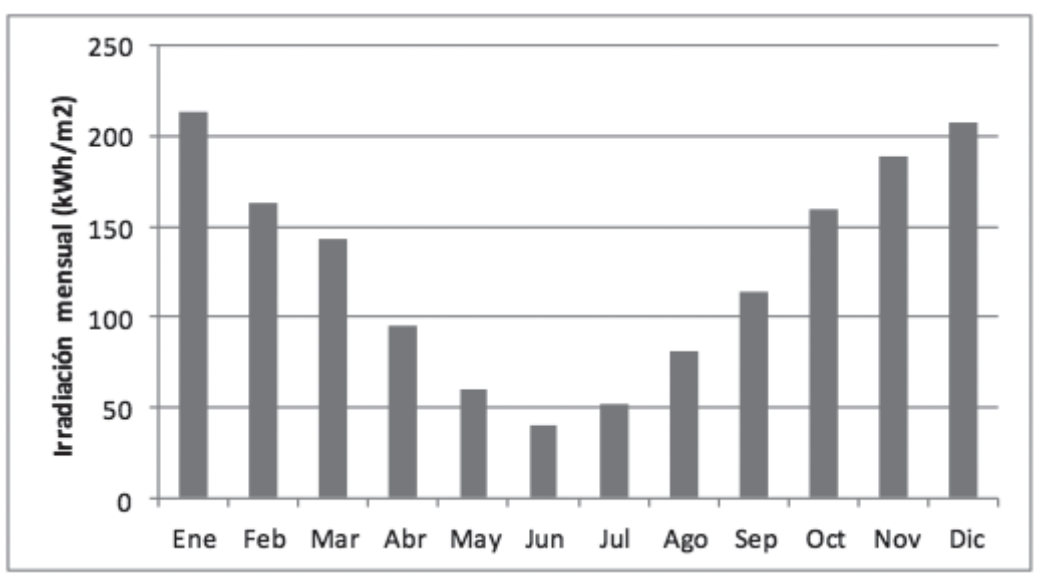

Fuente: CNE, PNUD y UTFSM, 2008.

Figura $N^{\circ} 3$

Radiación Solar promedio mensual sobre un plano vertical en Concepción, orientado $34.41^{\circ} \mathrm{NE}$, respeto al $\mathrm{N}$ geográfico

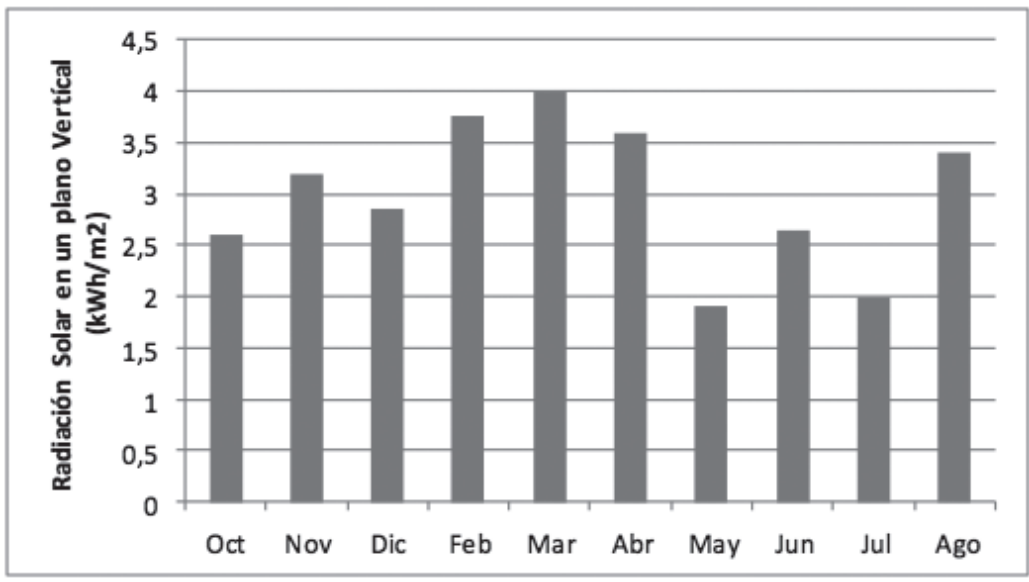

Fuente: Fissore \& Fonseca, 2007. 
curioso observar que en agosto (invierno) se alcanza un promedio diario de $3,4 \mathrm{kWh} / \mathrm{m}^{2}$, valor que supera a lo registrado durante la primavera anterior (octubre). Esto se debe al bajo ángulo de incidencia de radiación solar sobre la superficie. Debido a estas situaciones, es posible vaticinar que una correcta ubicación de los captadores solares permitiría un mayor aprovechamiento de la captación solar, generando importantes ahorros en el consumo energético durante el invierno, o bien en otra ubicación urbana.

\section{Elaboración del mapa energético solar de Concepción}

La construcción de los mapas energéticos se basa en la recopilación de la información necesaria para poder representar territorialmente los diferentes datos (consumos energéticos, tipologías, etcétera), que en algunos casos pueden desplegarse en distintos perfiles temporales.

Cuadro $N^{\circ} 1$

Esquema Metodológico

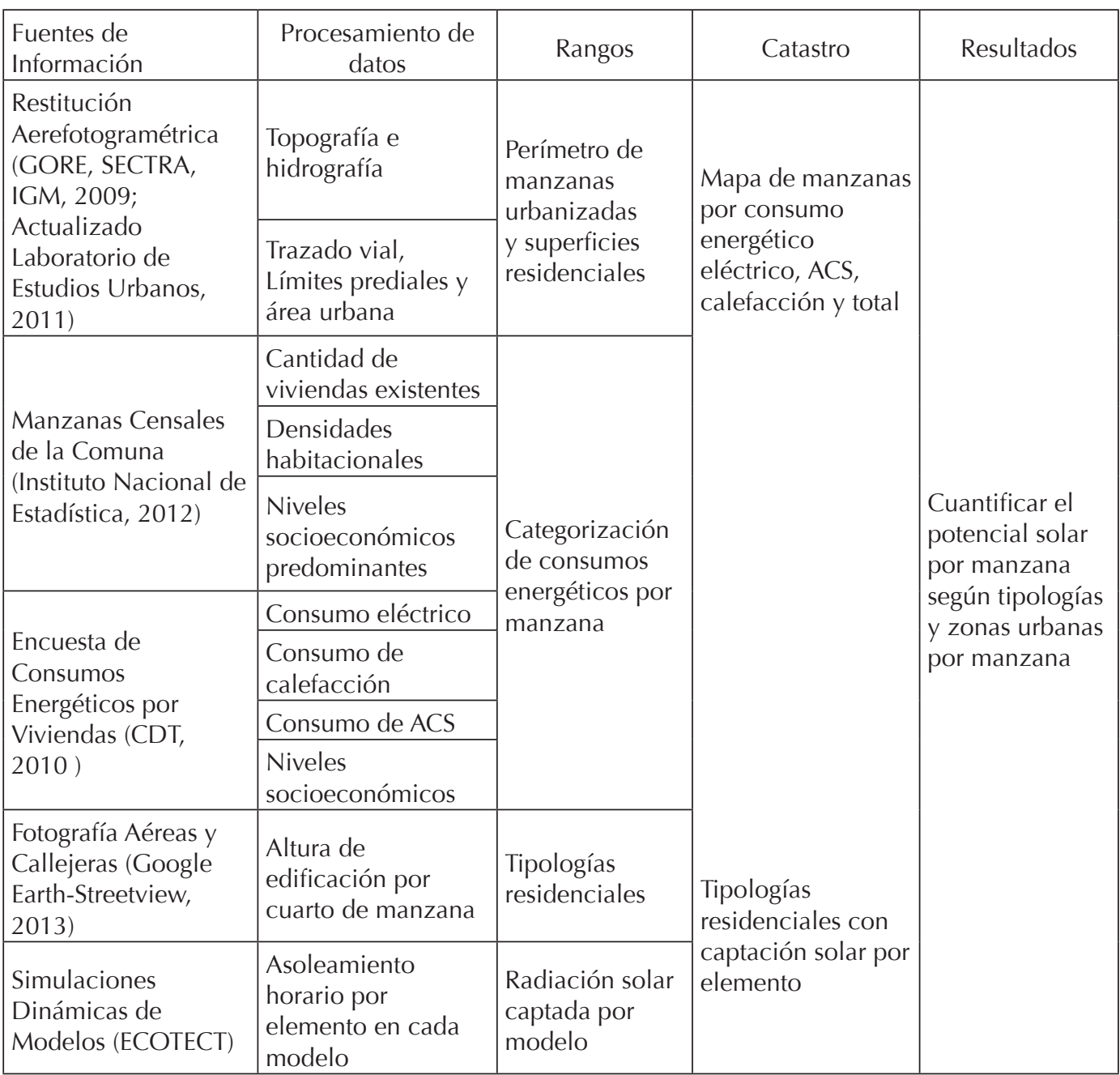

Fuente: Elaboración propia. 
Los consumos energéticos analizados son tres: Calefacción, Agua Caliente Sanitaria y Electricidad, todos según densidad residencial y nivel socioeconómico por manzana construida (equivalentes a una hectárea, $10.000 \mathrm{~m}^{2}$ ). La cantidad de consumo es extraída a partir de una encuesta realizada en la ciudad, donde se presentan distintos parámetros de medición, como cantidad de usuarios por vivienda, nivel socioeconómico, equipos utilizados, etcétera.

Para la calefacción, el análisis de consumo energético, se complementó con un estudio de demandas energéticas reales mediante simulaciones dinámicas de distintas tipologías residenciales construidas en la ciudad de Concepción. Estas viviendas fueron simuladas suponiendo una temperatura de confort para invierno de $18,5^{\circ} \mathrm{C}$ y con una ocupación de una familia tipo de 4 personas.

Para la creación de los mapas y evaluación del potencial solar del territorio, fue necesaria la integración de diversas fuentes de información (Cuadro $N^{\circ} 1$ ), provenientes en distintos formatos: fotografías, tablas, mapas, etc. Debido a esto se hace necesaria la organización de la información sobre el área de estudio, para generar una base de datos territorial operada a través de un Sistema de Información Geográfica (SIG) en software ArcMap 10.

Con la base de datos configurada, en función de la cantidad de capas necesarias para completar la información requerida, se procede al análisis del potencial solar, y así tener como resultados mapas y tablas que permitirán desarrollar estrategias de captación y aprovechamiento solar.

\section{Categorización de consumos energéticos}

Como base cartográfica se utiliza una restitución aerofotogrametría (Datum WGS84 Huso 18 Sur) de la provincia de Concepción, escala 1:10.000, realizada el año 2009 por el Gobierno Regional del Biobío, Secretaría de Transporte e Instituto Geográfico Militar, actualizado por el Laboratorio de Estudios Urbanos de la U. Bío-Bío el año 2011 y que integra el Plan Regulador Metropolitano y
Comunal del 2003. Esta base permite identificar niveles topográficos y cursos de agua, trazado vial, límites prediales y área urbana de la comuna de Concepción, implementado como archivo .shp en ArcMap. Luego se identifican los perímetros de manzanas urbanizadas, correspondientes a áreas residenciales, para así revisar el catastro público presentado por el Instituto Nacional de Estadísticas en convenio por MapCity (INE, 2013) que indica, entre otros, la cantidad de viviendas construidas y el grupo socioeconómico ${ }^{7}$ predominante por manzana censal (grupos de clasificación: ABC1, C2, C3, D). Estos datos son llevados al registro de perímetros residenciales de la base cartográfica, lo que permite calcular la densidad habitacional por manzana $\left(10.000 \mathrm{~m}^{2}\right)$. Las densidades se pueden diferenciar en cuatro rangos representativos de ocupación residencial, las cuales se combinan con grupos socioeconómicos predominantes para disponer de una caracterización de manzanas según densi$\mathrm{dad} /$ nivel.

Como ya se indicó, los datos de consumo energéticos son obtenidos a partir de encuestas realizadas el año 2010 para el estudio nacional de energía residencial (CDT, 2010), donde se consulta el gasto domiciliario, convertido a potencia calorífica por unidades habitacionales identificadas por comuna, tipología constructiva y nivel socioeconómico. Los consumos están diferenciados por gasto eléctrico, en calefacción y de agua caliente sanitaria. Las medias residenciales de consumos por nivel socioeconómico se calculan por densidad residencial obteniendo por tanto dieciséis categorías de gasto energético aplicables por manzana (Cuadro $N^{\circ} 2$ ).

\footnotetext{
7 Según la clasificación socioeconómica de Chile, correspondiente a un análisis estadístico de estratificación social, donde los criterios considerados involucran nivel de estudios alcanzados y nivel de riqueza. Abc 1, corresponde al nivel más alto, con mayores recursos, y por otro lado, D es el nivel socioeconómico más bajo.
} 

CONSUMO ENERGÉTICO Y CAPTACIÓN SOLAR EN EDIFICACIONES RESIDENCIALES DE CONCEPCIÓN, CHILE

Cuadro $\mathrm{N}^{\circ} 2$

Consumos energéticos anuales (MW/h ha) por niveles socioeconómicos y densidad residencial

\begin{tabular}{|l|r|r|r|r|}
\hline Densidad residencial & $\begin{array}{c}\text { Muy Baja } \\
\text { 1 a 15 viviendas } \\
\text { / hectárea }\end{array}$ & $\begin{array}{c}\text { Baja } \\
16 \text { a } 30 \text { viviendas } \\
\text { / hectárea }\end{array}$ & $\begin{array}{c}\text { Media } \\
31 \text { a } 75 \text { viviendas } \\
\text { / hectárea }\end{array}$ & $\begin{array}{c}\text { Alta } \\
76 \text { y más viviendas } \\
\text { / hectárea }\end{array}$ \\
\hline $\begin{array}{l}\text { Promedio de Viviendas } \\
\text { por Hectárea (unidades) }\end{array}$ & 10 & 24 & 60 & 100 \\
ABC1 & 390 & 936 & 1.200 & 2.000 \\
C2 & 200 & 480 & 720 & 1.200 \\
C3 & 180 & 432 & 660 & 1.100 \\
D & 130 & 312 & 480 & 800 \\
\hline
\end{tabular}

Fuente: Elaboración propia.

Esta distribución evidencia una progresión entre el nivel socioeconómico (por capacidad de pago) y la agrupación urbana. Para expresar más nítidamente esta incidencia en el catastro se definen cuatro categorías de consumos según las magnitudes presentadas, que son equivalentes en los distintos tipos de gastos (Cuadro $\mathrm{N}^{\circ} 3$ ).

Cuadro $\mathrm{N}^{\circ} 3$

Categorías de consumos (MW/h ha) a partir de los 16 indicadores

\begin{tabular}{|l|r|r|r|r|}
\hline Rango & $\begin{array}{r}\text { Consumo Energético } \\
\text { Total }\end{array}$ & $\begin{array}{c}\text { Consumo Energético } \\
\text { Eléctrico }\end{array}$ & $\begin{array}{c}\text { Consumo Energético } \\
\text { Calefacción }\end{array}$ & $\begin{array}{c}\text { Consumo Energético } \\
\text { ACS }\end{array}$ \\
\hline I & $130-400$ & $14-40$ & $110-299$ & $10-50$ \\
II & $401-700$ & $41-90$ & $300-500$ & $51-130$ \\
III & $701-1.099$ & $91-160$ & $501-800$ & $131-300$ \\
IV & $1.100-2.000$ & $161-280$ & $801-1000$ & $301-500$ \\
\hline
\end{tabular}

Fuente: Elaboración propia.

Estas categorías son implementadas en los perímetros residenciales de manzanas de la base cartográfica en SIG, para representar la distribución urbana del consumo energético habitacional en la comuna de Concepción, diferenciados según los distintos gastos consultados (Figura $N^{\circ} 4$ ).

Los mapas de consumo energético residencial en Concepción evidencian una concentración, con manzanas centrales de consumo cuatro veces superior a manzanas perimetrales que se extienden mayormente. Con interrupciones geográficas del área urbana e incrementos parciales en los sectores ocupados. La concentración se focaliza leve- mente al sur-oeste contiguo a un faldeo que agrupa alta densidad residencial de sectores medios-altos, y al centro cívico de servicios adyacente al oeste. Las distribuciones por tipo de gasto son similares, exceptuando el agua caliente sanitaria de menor concentración (pero mayor disparidad).

\section{Distribución de tipologías residenciales}

El catastro de tipologías residenciales, tiene por objetivo caracterizar las construcciones habitacionales y su distribución en la ciudad, para estimar capacidades de capta- 
Figura $\mathrm{N}^{\circ} 4$

Mapas por tipo de Consumo Energético en Comuna de Concepción

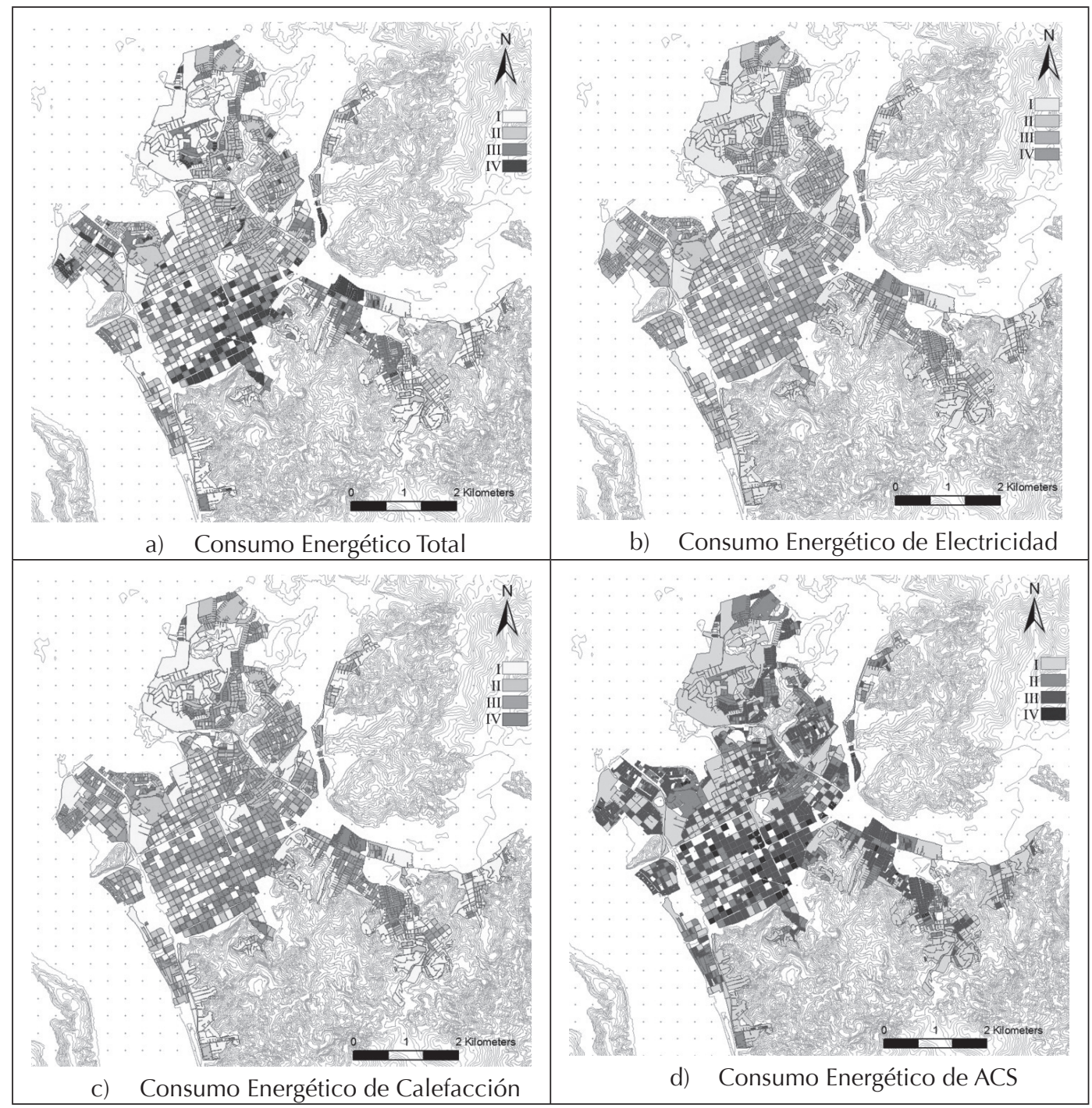

Fuente: Elaboración propia.

ción solar directa según áreas urbanas. Las edificaciones residenciales se constituyen en general por viviendas unifamiliares de baja altura (de 1 a 2 pisos) y propiedad individual del terreno, que conforman agrupaciones similares con densidades poblacionales reducidas (inferiores a 100 hab./ha), o por edificios multifamiliares (de departamentos) con mayor altura y propiedad colectiva del terreno, que generan sectores de mayor densidad poblacional (sobre 100 hab./ha). Esta clasificación morfológica es la manera de agrupar distintas tipologías residenciales en grupos de acuerdo al predominio de cada tipología por manzana, o cuarto de manzana, identificada, donde se combina mucha variedad de edificación. $\mathrm{Si}$ bien las edificaciones residenciales construidas en Concepción se consideran como diversas tipológicamente, estas maneras de agrupar permitirán tener un catastro de las tipologías morfológicas más preponderantes. 

CONSUMO ENERGÉTICO Y CAPTACIÓN SOLAR EN EDIFICACIONES RESIDENCIALES DE CONCEPCIÓN, CHILE

En Chile, de manera similar a otros países latinoamericanos o en desarrollo, las viviendas unifamiliares constituyen la tipología predominante con $87 \%$ del total (MINVU, 2010). Aunque en las áreas metropolitanas la proporción de edificios departamentos se incrementa, hasta igualar ambas tipologías como en el caso de comunas centrales como Concepción (DOM, 2013). De acuerdo a la ocupación urbana, las viviendas unifamiliares se distinguen en edificaciones de mayor tamaño y menor densidad (usualmente de niveles socioeconómicos medios-altos), y otras de menor tamaño (aunque similar altura) y densidad más variable, que alojan sectores medios y bajos. Los asentamientos precarios o irregulares han sido reducidos sustancialmente en el país, y en esta comuna se presentan en sectores reducidos y temporales. Entre los edificios multifamiliares, se diferencian los inferiores a cinco pisos (por la normativa vigente no requieren ascensores y por tanto suelen ser más extensos) denominados "bloques", y los mayores, que por rentabilidad de inversión oscilan entre diez a veinte pisos, llamados "torres", y subsecuentemente generan mayor densidad y se localizan en lugares más céntricos o de alta renta. Por lo que, para analizar la edificación se establecieron cuatro tipos residenciales, primeramente según altura, y en las construcciones bajas, se diferenció también por densidad (Cuadro $\mathrm{N}^{\circ} 4$ ).

\section{Cuadro $\mathrm{N}^{\circ} 4$}

Tipos residenciales según altura de edificación y densidad estimada

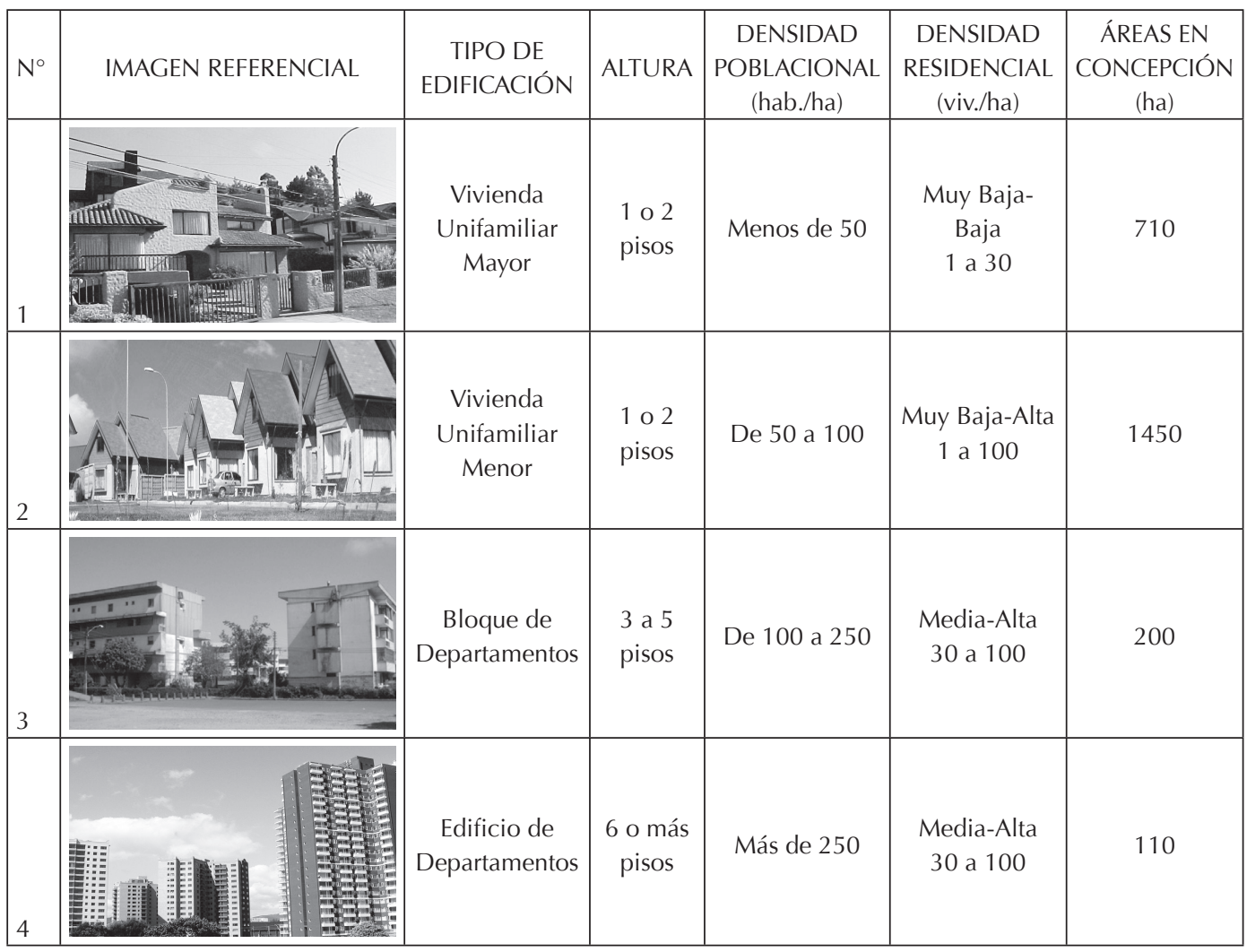

Fuente: Elaboración propia.

La identificación de las tipologías se realizó mediante foto-interpretación aérea (basada en Google Earth v.7.1.1) y verificación por panorámicas peatonales (de Google StreetView v7.2.0). En algunos casos también con visitas a terreno. Los criterios empleados en 
el proceso de foto-interpretación, para determinar las tipologías, fueron los siguientes:

- Diferencias regulares de tonalidad que expresan formas ortogonales.

- Áreas oscuras laterales que expresan sombras de volúmenes constructivos.

- Tamaño proporcional a edificaciones (según referencias visuales)

- Distribución en tramas equivalentes a conjuntos o tejidos urbanos.

- Complemento con trazados de vías y áreas verdes.

De este modo, las manzanas residenciales con edificaciones menores, más distanciadas y con menores sombras se identificaron como tipo 1, las más próximas y usualmente de menor dimensión y sombras parciales como tipo 2, edificaciones más extensas y de sombras medias como tipo 3, y finalmente las formas menores, pero con sombras más extensas como tipo 4. En este proceso se verifica con las fotos peatonales y visitas, evidenciando coherencia en la localización urbana por sectores (Figura $\mathrm{N}^{\circ} 5$ ).

El mapa de tipologías advierte una distribución también concéntrica con algunas singularidades periféricas. Expresando un predominio territorial de la vivienda unifamiliar menor (tipo 2) y una localización central

Figura $N^{\circ} 5$

Tipologías residenciales en la comuna de Concepción

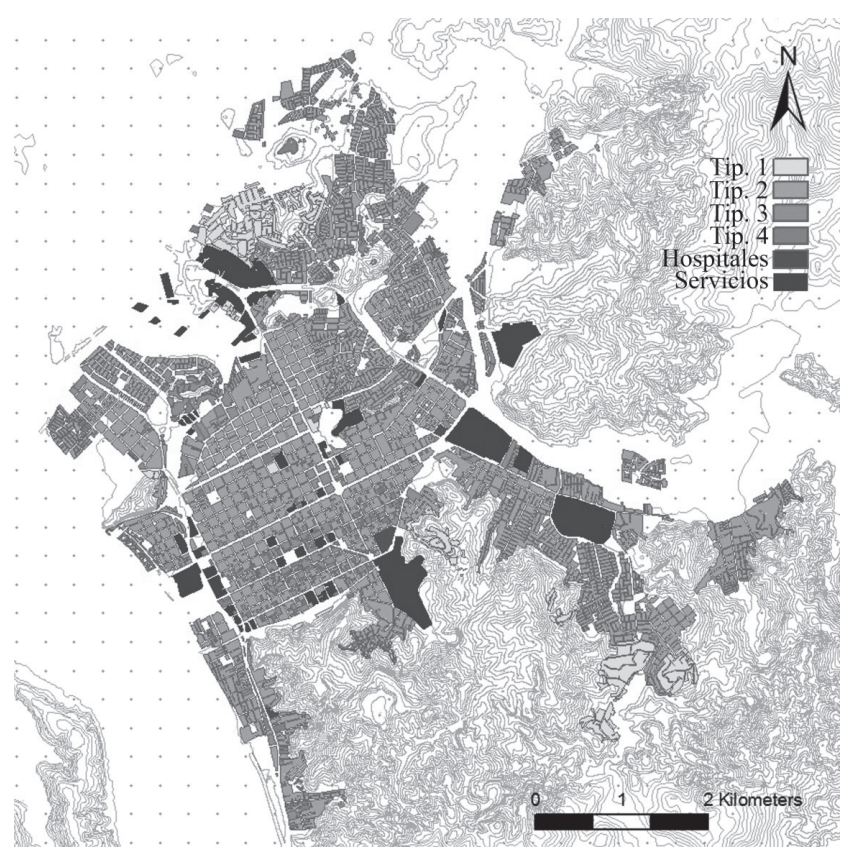

Fuente: Elaboración propia.

de la edificación en altura (tipo 3 y 4), que se combina muchas veces con ocupación comercial y de servicios (reconocidas como manzanas de baja densidad residencial). Se identifican sectores con vivienda unifamiliar mayor (tipo 1) en la periferia, y en el anillo intermedio con residencias en altura, siendo localizada en tramas de mayor irregularidad, debido a nuevos asentamientos de crecimiento de la ciudad de Concepción. Este patrón de edificación es similar en la mayoría de las ciudades chilenas.

\section{Análisis de captación solar de las tipologías residenciales}

La captación solar en cada tipología se analizó por simulaciones de algunos modelos de edificación, seleccionados por las fotogra- 
fías aéreas y verificación en terreno (Cuadros $\mathrm{N}^{\circ} 5$ y $\mathrm{N}^{\circ} 6$ ), con sus respectivas características reales, como su orientación con respecto al norte, que no en todos los casos era la misma, ni tampoco la más favorable. Cada modelo se simuló en un software de simulación energético ambiental (ECOTECT de Autodesk), con base climática IWEC, determinando el asoleamiento recibido en cada plano de la configuración para fechas extremas del calendario solar (equinoccio y solsticio de verano e invierno), considerando valores que han sido medidos por estaciones fijas en la ciudad durante períodos prolongados, permitiendo llegar a promedios estadística- mente confiables. Se contabilizó la cantidad de horas de asoleamiento recibido sobre el $50 \%$ de la superficie, considerando sombreamientos propios o arrojados por cierros (no se consideró vegetación) o edificaciones vecinas (modelando el conjunto). Luego se efectúa una interpolación para el total de horas anuales y una cuantificación de la energía recibida en cada plano según azimut e inclinación de acuerdo a los valores de radiación local. Finalmente los distintos planos de cada componente (techos, muros y sitio) son sumados en las viviendas individuales o divididos en las viviendas colectivas, para determinar la magnitud anual acumulada por unidad.

Cuadro $\mathrm{N}^{\circ} 5$

Descripción de Modelos Residenciales

\begin{tabular}{|c|c|c|c|c|c|c|}
\hline Tipología & Modelos & Adosamiento & Ancho/Largo & Pisos & Sup.total & Sitio \\
\hline \multirow{2}{*}{1} & $1 \mathrm{a}$ & Aisl. Rectang. & $8 \times 10$ & 2 & $140 \mathrm{~m}^{2}$ & $18 \times 25$ \\
\hline & $1 \mathrm{~b}$ & Ais. en Ele & $11 \times 11 \times 6 \times 6$ & 2 & $140 \mathrm{~m}^{2}$ & $18 \times 25$ \\
\hline \multirow{4}{*}{2} & $2 \mathrm{a}$ & Continua & $7 \times 7$ & 1 & $50 \mathrm{~m}^{2}$ & $7 \times 15$ \\
\hline & 2b1 & Pareada N & $7 \times 10$ & 1 & $70 \mathrm{~m}^{2}$ & $10 \times 20$ \\
\hline & $2 \mathrm{~b} 2$ & Pareada S & $7 \times 10$ & 1 & $70 \mathrm{~m}^{2}$ & $10 \times 20$ \\
\hline & $2 c$ & Aislada & $6 \times 8$ & 2 & $90 \mathrm{~m}^{2}$ & $12 \times 21$ \\
\hline \multirow{4}{*}{3} & $3 a$ & Depto. Esq.NE & $6 \times 9$ & 1 & $54 \mathrm{~m}^{2}$ & $50 \times 50$ \\
\hline & $3 b$ & Dep. Medio N & $6 \times 9$ & 1 & $54 \mathrm{~m}^{2}$ & $50 \times 50$ \\
\hline & $3 c$ & Dep. Medio S & $6 \times 9$ & 1 & $54 \mathrm{~m}^{2}$ & $50 \times 50$ \\
\hline & $3 d$ & Depto. Esq. SW & $6 \times 9$ & 1 & $54 \mathrm{~m}^{2}$ & $50 \times 50$ \\
\hline \multirow{4}{*}{4} & $4 \mathrm{a}$ & Depto. Esq.NE & $7 \times 11$ & 1 & $77 \mathrm{~m}^{2}$ & $70 \times 70$ \\
\hline & $4 b$ & Dep. Medio N & $7 \times 11$ & 1 & $77 \mathrm{~m}^{2}$ & $70 \times 70$ \\
\hline & $4 c$ & Dep. Medio S & $7 \times 11$ & 1 & $77 \mathrm{~m}^{2}$ & $70 \times 70$ \\
\hline & $4 d$ & Depto. Esq. SW & $7 \times 11$ & 1 & $77 \mathrm{~m}^{2}$ & $70 \times 70$ \\
\hline
\end{tabular}

Fuente: Elaboración propia.

En la vivienda mayor (tipo 1), que presenta una relevante diversidad de formas, dimensiones y distribuciones, se realizaron dos modelos de tamaño similar a la superficie que se construye usualmente $\left(140 \mathrm{~m}^{2}\right)$. Aplicando diferentes configuraciones geométricas (rectangular y en "L"), se revisan incidencia de sombras propias, ya que usualmente son construcciones independientes y alejadas que no le afectan mucho las edificaciones vecinas. En las viviendas menores (tipo 2), se modelaron cuatro alternativas para recoger diferencias de agrupación y altura que se consideran regularmente en estas construcciones, como se advierte en el mismo extracto fotográfico. En magnitudes de 50 a $90 \mathrm{~m}^{2}$ de superficie que corresponde al tramo central de edificación residencial en el país, en una conformación continua (adosada por dos costados) de un piso, otra conformación pareada por un solo costado también en un piso, estudiando las dos viviendas correspon- 
Cuadro $N^{\circ} 6$

Fotografía referencial y Modelos simulados

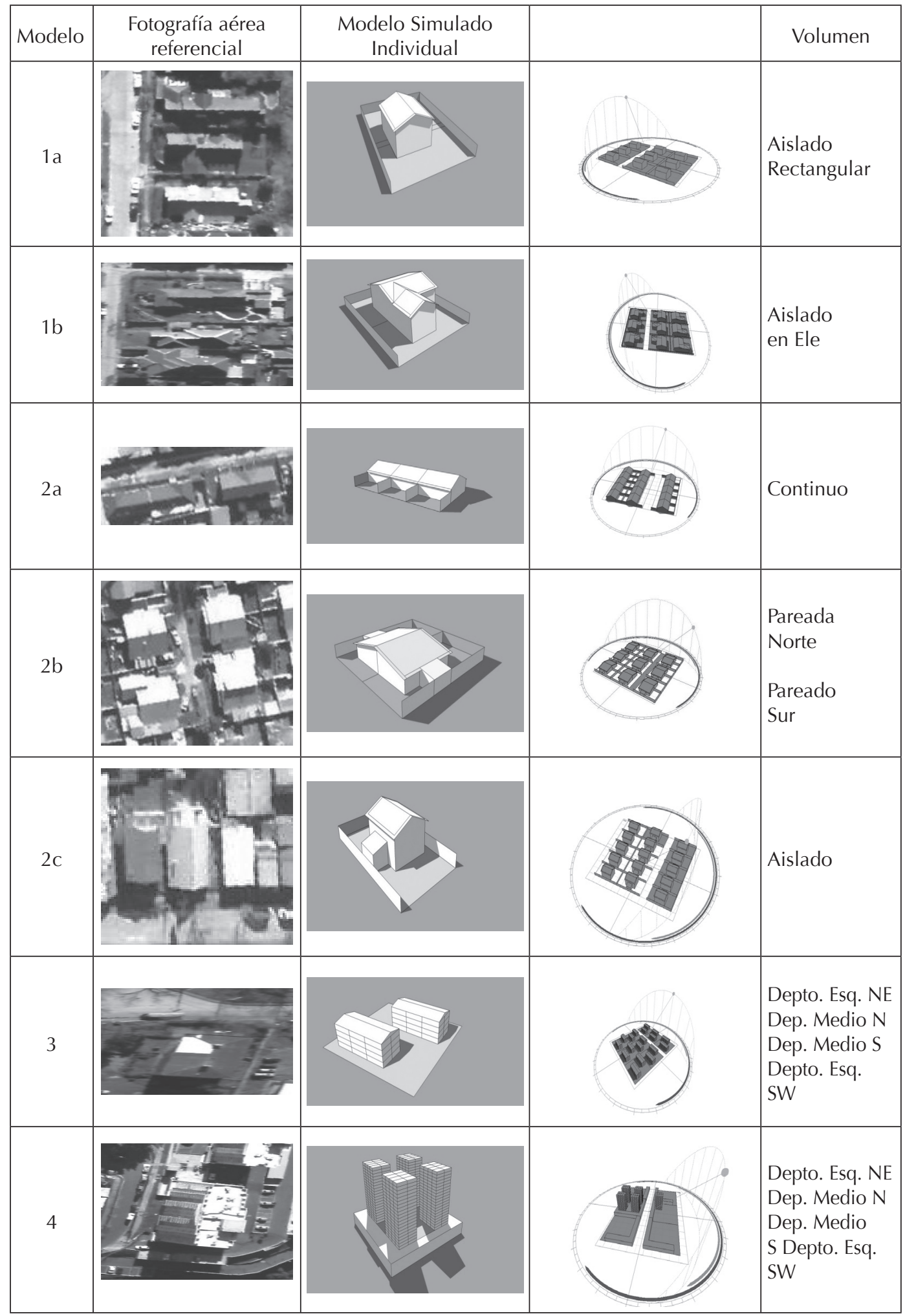

Fuente: Elaboración propia. 
dientes, y una tercera conformación aislada en dos pisos, que suele elevarse por su individualidad en sitios de similar dimensión. Se consideraron modelos simétricos, por lo que no se revisaron distintas orientaciones que suelen tener incidencias similares, excepto en las pareadas Norte-Sur que se revisaron por separado debido a sus diferencias significativas, en especial con techumbre de una inclinación. En los edificios residenciales en altura, en ambos casos (Tipo 3 y 4) se analizaron departamentos medios en las dos orientaciones extremas de asoleamiento (Norte y Sur), $y$ en las esquinas enfrentadas (NE - SW). No se revisaron distintas alturas, debido a que se advirtió escasa incidencia de esta variación. Los sitios y techos se consideraron de manera colectiva, dividiendo luego proporción correspondiente por unidad, considerando 24 y 96 departamentos respectivamente en cada caso.

Las magnitudes de captación solar obtenida por conjuntos de componentes en cada modelo (Figura $\mathrm{N}^{\circ}$ 6), presentan una diferencia sustancial y regular entre las tipologías. Las viviendas unifamiliares revelan potenciales globales que superan los $100.000 \mathrm{~kW} / \mathrm{h}$ anuales, alcanzando solo en las techumbres sobre $60.000 \mathrm{~kW} / \mathrm{h}$ año. Mientras los departamentos presentan magnitudes entre 15.000 a $50.000 \mathrm{~kW} / \mathrm{h}$ año. Evidenciando una relación inversa con la altura y densidad urbana, que se reconoce también entre las viviendas unifamiliares. Además de oscilaciones relevantes entre las configuraciones de viviendas y orientaciones de los departamentos, aunque dentro de los rangos de cada tipología.

Se debe destacar que la captación en las viviendas individuales expresan magnitudes cercanas a los consumos detectados (entre 8.000 y $40.000 \mathrm{kWh} / \mathrm{año}$ de consumo energético en tipologías residenciales), incluso desde uno solo de los componentes, principalmente la techumbre en todos los modelos. Considerando rendimientos de sistemas térmicos (aproximadamente 50\%) o fotovoltaicos (cercanos al 15\%) y la instalación de sistemas completos, con almacenamiento y conversión adecuadas (CDT, 2013). En las viviendas mayores se podrían considerar tanto en cubiertas, muros o partes exteriores. En las viviendas menores fundamentalmente en las techumbres. En los departamentos se expresan capacidades relevantes en las techumbres compartidas y en las fachadas (especialmente los orientados al norte), que pueden participar en partes relevantes de la demanda.

La mayor captación solar de las viviendas unifamiliares respecto a las multifamiliares en

Figura $\mathrm{N}^{\circ} 6$

Gráfico de Resultados de Captación Solar por Modelo de Vivienda (kW/h año)

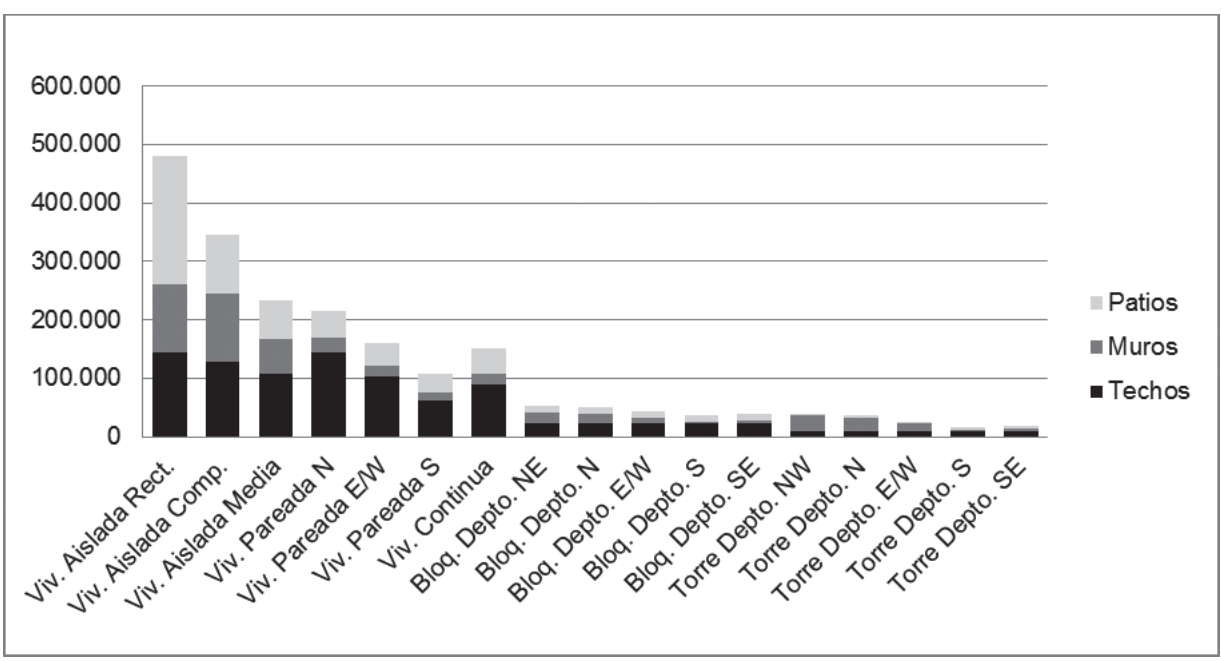

Fuente: Elaboración propia. 
altura, es similar a la diferencia en consumos, que aumenta en las construcciones aisladas por su mayor disipación energética sobre las edificaciones agrupadas (Bustamante et al., 2009). Lo que se expresa en Cuadro $N^{\circ} 2$ si se consideran valores unitarios por vivienda, pero que se incrementa por la densidad residencial. Esta relación permite equiparar las diferencias de eficiencia energética de las tipologías habitacionales, aunque es menor a las magnitudes de captación, que sugieren diferentes estrategias de recolección. En las viviendas unifamiliares parece más pertinente utilizar las techumbres, y en los edificios multifamiliares las fachadas orientados al norte.

\section{Potencial solar urbano}

El potencial solar urbano se puede considerar como la diferencia por unidades o sectores entre la captación y el consumo individual, es decir lo que pueden aportar las edificaciones además de cubrir sus propias necesidades, con el fin de integrar provisión local entre distintas zonas. Considerando la captación global ponderada por los rendimientos de sistemas específicos de recolección, este potencial se puede estimar por manzanas a partir de las medias de captación de cada tipología predominante en relación a las categorías de consumo determinadas. Si el valor es mayor de uno, implica que las tipologías de esa manzana pueden cubrir sus necesidades si utilizan la captación solar de todos sus componentes, y además contribuir con una diferencia a la red. Si el valor es inferior significa que pueden asumir parcialmente sus propios consumos y recibir del sistema general.

Cuadro $\mathrm{N}^{\circ} 7$

Potencial Solar Urbano por manzana en relación a las medias de las categorías de consumos

\begin{tabular}{|c|r|r|r|r|r|r|}
\hline Tipos & $\begin{array}{c}\text { Densidad } \\
\text { residencial } \\
\text { (Viv./ha) }\end{array}$ & $\begin{array}{c}\text { Captación } \\
\text { media total (MW/h } \\
\text { ha año *15\%) }\end{array}$ & $\begin{array}{c}\text { Categoría } \\
\text { de } \\
\text { Consumo }\end{array}$ & $\begin{array}{c}\text { Consumo } \\
\text { total } \\
\text { (MW/Ha año })\end{array}$ & $\begin{array}{c}\text { Potencial } \\
\text { solar } \\
\text { urbano }\end{array}$ & Rango \\
\hline 1 & 1 a 15 & 620 & I & $130-400$ & 2,48 & Alta \\
1 & 16 a 30 & 1.487 & II & $401-700$ & 2,70 & Alta \\
2 & 1 a 15 & 296 & I & $130-400$ & 1,12 & Media \\
2 & 16 a 30 & II & $401-700$ & 1,29 & Media \\
2 & 31 a 75 & 1.777 & III & $701-1.000$ & 2,09 & Media-Alta \\
2 & 76 y mas & 2.962 & IV & $1.001-2.000$ & 1,97 & Media-Alta \\
3 & 31 a 75 & 375 & III & $701-1.000$ & 0,44 & Baja \\
3 & 76 y mas & 624 & IV & $1.001-2.000$ & 0,42 & Baja \\
4 & 76 y mas & 514 & IV & $1.001-2.000$ & 0,34 & Baja \\
\hline
\end{tabular}

Fuente: Elaboración propia.

En las tipologías identificadas para la comuna de Concepción, según las distintas densidades residenciales, se puede estimar una media de captación solar por manzana ponderada por rendimiento regular de equipos fotovoltaicos (15\%), y al ser relacionadas con las medias de las categorías de consumos, se puede obtener una magnitud de potencial solar urbano por manzana (Cuadro $N^{\circ} 7$ ). Esta estimación revela cuatro rangos fundamentales de potencial solar urbano en la comuna, alto (sobre el doble de captación) para las viviendas mayores, que presentan altos consumos pero también captaciones relevantes que pueden aportar al sistema; medio-alto de las viviendas menores en baja densidad y medio en mayor densidad, en ambos casos cubriendo sus requerimientos y otorgando contribución, lo que es relevante porque esta tipología es la más extensa y alberga la mayor parte de la población. En los departamentos, tanto de bloque, como de torres, se revela un 

CONSUMO ENERGÉTICO Y CAPTACIÓN SOLAR EN EDIFICACIONES RESIDENCIALES DE CONCEPCIÓN, CHILE

potencial bajo, aunque suficiente para cubrir cerca de la mitad de los consumos (con estra- tegias diferentes), y que pueden recibir de las restantes tipologías.

Figura $\mathrm{N}^{\circ} 7$

Mapa de Potenciales Solares Urbanos en la comuna de Concepción

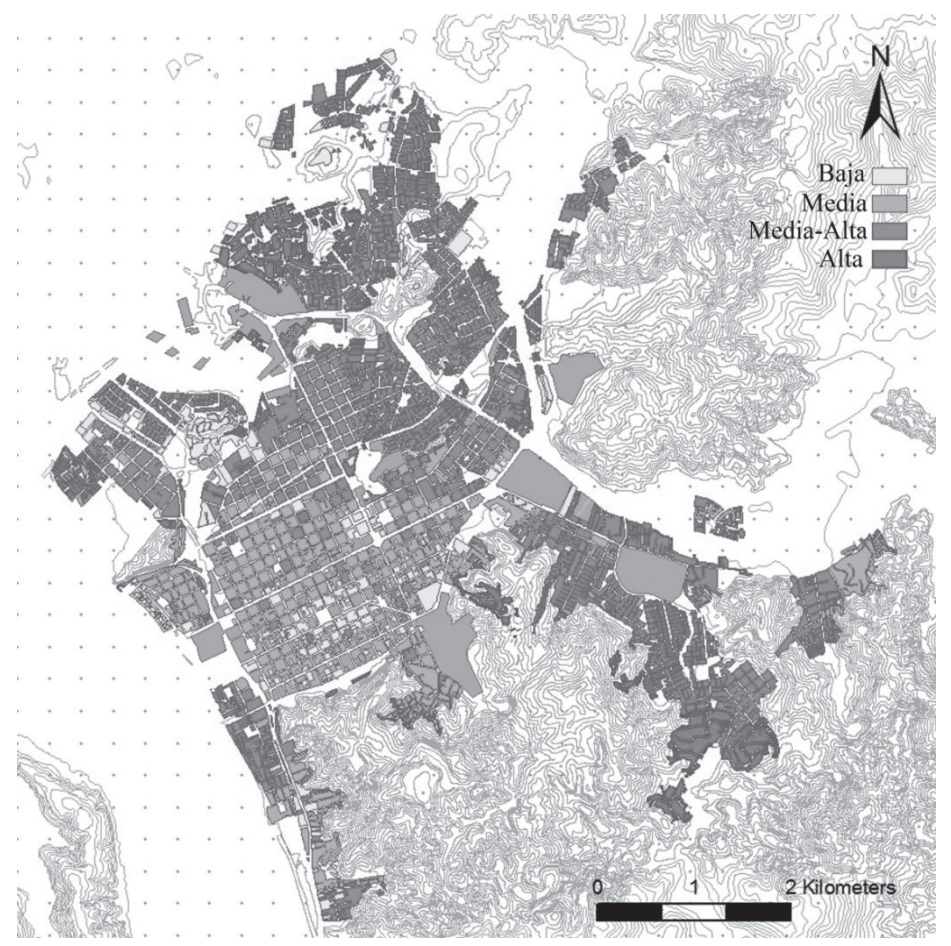

Fuente: Elaboración propia.

Estos resultados expresan que el potencial urbano de la energía solar residencial en los sectores de mayor densidad es más bajo que en los de menor densidad (donde el nivel socioeconómico es más alto, y por ende el consumo energético también), caracterizados por viviendas unifamiliares. Esto es consecuencia, entre otras cosas, de la menor superficie expuesta por unidad habitacional y por la mayor incidencia de los conos de sombra. Por el contrario, en el caso de las viviendas en extensión el potencial es mayor, por la mayor captación solar disponible.

\section{Conclusiones}

El catastro de consumos energéticos y captación solar de las edificaciones residenciales realizado para la comuna de Concepción evidencia en primer lugar una estrategia de análisis urbano para promover la utiliza- ción de energías renovables en las ciudades. Basada es encuestas de gastos domiciliarios, niveles socioeconómicos, fotos aéreas, tipologías habitacionales y simulación de modelos, implementado en un SIG. La cartografía realizada permite determinar zonas urbanas con gastos relevantes, pero también posibilidades sustanciales de aprovechamiento solar. Con detalle por manzana (o cuartos de manzanas), aunque con variaciones particulares por edificación que deben revisarse individualmente. Sugiriendo una metodología complementaria a los procesos basados en registros centrales y levantamientos tridimensionales, como LIDAR (Levinson, 2009). Por lo que brinda un proceso pertinente para estimar potenciales energéticos renovables para áreas urbanas, aunque de precisiones mayores.

En el registro de consumos residenciales realizado en Concepción se observa que se 
incrementan sustancialmente (se triplican), según el rango de niveles socioeconómicos predominantes por manzana (Cuadro $\mathrm{N}^{\circ}$ 2). Sin embargo la distinción por densidad habitacional es mayor, debido a grandes diferencias de concentración por tipologías constructivas, por lo que los mapas de consumos reflejan mayormente el agrupamiento de las viviendas, con incidencias similares por tipos de servicios (Figura $N^{\circ} 4$ ). Presentando una distribución urbana principalmente centralizada, aunque con singularidades perimetrales por el entorno topográfico.

Lo opuesto ocurre con la captación solar de las edificaciones existentes, ya que según las simulaciones de algunos modelos representativos, se presenta mayor posibilidad de aprovechamiento en las zonas periféricas de la comuna, es decir donde el consumo energético es menor (Figura $N^{\circ} 7$ ). En cambio, en las áreas centrales, que más abastecimiento de energía consumen, se encuentran tipologías de menor captación solar. Aunque se debe considerar que poseen demandas individuales inferiores, debido a que las tipologías más densas presentan mayor eficiencia energética, y además esta diferencia puede ser abordada con distintas estrategias de recolección (Redweik, 2013).

Estos resultados sugieren revisar la ventaja atribuida al modelo de crecimiento compacto de las ciudades, desde la perspectiva de la sustentabilidad. Los atributos de este modelo, como el menor consumo de suelo, mejor cobertura de la infraestructura y transporte, entre otros, debieran formar parte de una matriz más integral de análisis, que también considere el consumo y potencial solar, con lo cual se pueden definir densidades de equilibrio que garanticen un ciclo más coherente entre la concentración habitacional, las tipologías y el aprovechamiento solar.

También, se puede inferir que en la ciudad de Concepción, los sectores de alta densidad presentan una menor captación solar, y por otro lado los sectores con baja densidad presentan un sobrepotencial. Esto permitiría suponer que mediante una transferencia entre los sectores se lograría un equilibrio en la matriz energética solar de la ciudad. Esta posibilidad, permitiría aproximarse al modelo de equilibrio urbano, en el cual una ciudad que promueve un modelo compacto, para mejorar los indica- dores de sustentabilidad, debe necesariamente contar con sectores urbanos de densidad mixta y/o de baja densidad. En los cuales sea posible contar con las condiciones necesarias para captar la energía potencial que cubra el consumo total de la ciudad, especialmente de los sectores más desventajados como los de mayor densidad habitacional.

Lo anterior parece confirmar el concepto de diversidad urbana, como un factor determinante de la sustentabilidad de la ciudad. Considerando el principio que un mayor abanico de tipologías, ofrece flexibilidad, adaptabilidad y mayor capacidad de opciones de provisión energética.

De este modo la elaboración de catastros zonales en base a informaciones individuales permite sugerir estrategias diferenciadas por áreas y tipologías correspondientes, aunque indudablemente una mayor precisión aporta dimensionamientos específicos por edificación y probablemente también distribuciones vecinales y temporales. En este sentido son desafíos relevantes, especialmente en las ciudades en desarrollo, avanzar en la sistematización de información y procedimientos para caracterizar de manera detallada los consumos energéticos y potenciales solares. La experiencia desarrollada permite ejemplificar procesos y motivar programas para abordar los desafíos ambientales de las ciudades.

\section{Referencias bibliográficas}

ABERG, M.; WIDÉN, J. \& HENNING, D. Sensitivity of district heating system operation to heat demand reductions and electricity price variations: A Swedish example. Energy, 2012, N41, p. 525-540.

ANHEIM SOLAR MAP (ASM). Disponible en Internet: http://anaheim.solarmap.org

BALBAO, M.; JORDÁN, R. y SIMIONI, D. La ciudad inclusiva. Santiago de Chile: Naciones Unidas, 2003.

BORJA, J. El gobierno del territorio de las ciudades latinoamericanas. Instituciones y Desarrollo, 2001, № 8-9, p. 83-142.

BUNJE, P.; BURNS, P.; CHESTER, M.; CIRCELLA, G.; FERREL, J.; FLAMING, D.; 

CONSUMO ENERGÉTICO Y CAPTACIÓN SOLAR EN EDIFICACIONES RESIDENCIALES DE CONCEPCIÓN, CHILE

MCCOY, M.; MURPHY, S. \& PINCETL, S. Methodology to Establish Regional Energy Baselines. Los Ángeles: California Energy Commission \& University of California, 2012.

BUSTAMANTE, W.; CEPEDA, R.; MARTÍNEZ, P. y SANTA MARÍA, H. Eficiencia energética en vivienda social: un desafío posible. Camino al Bicentenario, Propuestas para Chile. Santiago de Chile: Concurso Políticas Públicas, 2009.

CASTILLO, G. y MALDONADO, P. Situación de la Energía en Chile. Desafíos para la Sustentabilidad. Santiago de Chile: Programa Chile Sustentable, 2004.

CITY OF BERKELEY (CB). Berkeley SolarMap. Disponible en Internet: http://berkeley. solarmap.org/solarmap_v4.html

CALIFORNIA CENTER FOR SUSTAINABLE COMMUNITIES (CCSC). Electricity Map of Los Angeles. Disponible en internet: http:// sustainablecommunities.environment.ucla. edu/map/

CCTP. Chile necesita una gran reforma energética. Propuestas de la Comisión Ciudadana Técnico-Parlamentaria para la Transición hacia un Desarrollo Eléctrico Limpio, Seguro, Sustentable y Justo. Santiago de Chile: Comité Editorial Comisión Ciudadana-TécnicoParlamentaria para la Política y la Matriz Eléctrica, 2011.

CDT. Diseño y Dimensionamiento de Sistemas Solares Fotovoltaicos - conectados a Red. Santiago de Chile: CDT, 2001.

CORPORACIÓN DE DESARROLLO TECNOLÓGIO (CDT). Estudio de usos finales y curva de oferta de la conservación de la energía en el sector residencial. Santiago de Chile: CDT, 2010.

COMISIÓN NACIONAL DE ENERGÍA/ PROGRAMA DE NACIONES UNIDAS PARA EL DESARROLLO (PNUD) Y UNIVERSIDAD TÉCNICA FEDERICO SANTA MARÍA. Irradiancia solar en territorios de la República de Chile. Santiago de Chile: Proyecto CHI/00/ G32, 2008.

CANADIAN URBAN INSTITUTE (CUI). City of Guelph Integrated Energy Mapping
Strategy of 2010. Disponible en Internet: http://www.canurb.com/research-projects/ energy-mapping.htm

DACHFLÄCHENEIGNUNG FÜR SOLARANLAGEN (DS). Disponible en Internet: http://geo.gkd-el.de/website/solar/viewer.htm

DÍAZ, A. Mapas de radiación y soleamiento de ciudades. Disponible en internet: http://www.huellasolar.com

DIRECCIÓN DE OBRAS MUNICPALES (DOM). Información Geográfica Comunal, 2013. Concepción. Disponible en internet: http://200.68.12.118/SigDom_comps/SigDom.phtml

DIRECCIÓN METEOROLÓGICA DE CHILE. Disponible en Internet: http://www.meteochile.gob.cl/

EMDER SOLARKASTER (ES). Disponible en Internet: http://gis.stadtwerke-emden.de/ Solarkataster/solarkataster

FISSORE, A. La Realidad Energética en el Sector Residencial de la Región del Bío-Bío. Santiago de Chile: Alianza de Energía y Clima de las Américas, 2009.

FISSORE, A. \& FONSECA, N. Measurement results and experimental analysis study of the termal balance of a window. Building and Environment, 2007, № 42, p. 3570-3581.

GASTLI, A. \& CHARABI, Y. Solar electricity prospects in Oman using GIS-based solar radiation maps. Renewable and Sustainable Energy Reviews, 2010, N¹4, p. 790-797.

GILMOUR, B.; WARREN, J.; MARGEM, K.; MOLINA, J.; MYRANS, I.; CARR, C.; GERAGHTY, S. \& SULLIVAN, T. Integrated Energy Mapping for Ontario Communities (IEMOC): City of Guelph Energy Density Mapping Strategy Team. Toronto: Canadian Urban Institute, 2010

GRAUTHOFF, M.; JANSSEN, U.; FERNANDES, J. \& POLIS. Identification and mobilization of solar potentials via local strategies. Guidelines based on the experiences of the pilot actions in Lisbon, Lyon, Malmö, Munich, Paris and Vitoria-Gasteiz. Paris: InteIligent Energy Europe and POLIS, 2012. 
HOWARD, B.; PARSHALL, L.; THOMPSON, J.; HAMMER, S.; DICKINSON, J. \& MODIA, V. Spatial distribution of urban building energy consumption by end use. Energy and Buildings, 2012, $\mathrm{N}^{\circ} 45$, p. 141-151.

INTERNATIONAL ENERGY AGENCY (IEA). Cities, Towns and Renewable Energy: Yes In My Front Yard. Paris: International Energy Agency, 2009.

INSTITUTO NACIONAL DE ESTADÍSTICAS (INE). Chile: División Político-Administrativa y Censal 2001. Santiago de Chile: Instituto Nacional de Estadísticas, 2002.

INSTITUTO NACIONAL DE ESTADÍSTICAS (INE). Plataforma de Navegación según datos censales. Disponible en Internet: http://www. http://www.ine.cl/mapcity/

IZQUIERDO, S.; RODRIGUES, M. \& FUEYO, N. A method for estimating the geographical distribution of the available roof surface area for large-scale photovoltaic energy-potential evaluations. Solar Energy, 2008, $\mathrm{N}^{\circ}$ 82, p. 929-939.

JAKUBIEC, A. \& REINHART, C. A method for predicting city-wide electricity gains from photovoltaic panels based on LiDAR and GIS data combined with hourly Daysim simulations. Solar Energy, 2013, N `93, p. 127-143.

JANJAI, S.; LAKSANABOONSONG, J.; NUNEZ, M. \& THONGSATHITYA, A. Development of a method generating operational solar radiation maps from satellite data for a tropical environment. Solar Energy, 2005, No 78, p. 739-751.

LA COUNTY (LAC). Disponible en internet: http://solarmap.lacounty.gov/

LEVINSON, R.; AKBARI, H.; POMERANTZ, M. \& GUPTA, S. Solar access of residential rooftops in four California cities. Solar Energy, 2009, № 83, p. 2120-2135.

LUKAC, N. \& ZALIK, B. GPU-based roofs' solar potential estimation using LiDAR data. Computers \& Geosciences, 2013, N ${ }^{\circ}$ 52, p. 34-41.
LUND, P. Large-scale urban renewable electricity schemes - Integration and interfacing Aspects. Energy Conversion and Management, 2012, №63, p. 162-172.

LUND, P. Fast market penetration of energy technologies in retrospect with application to clean energy futures. Applied Energy, 2010, No 87, p. 3575-3583.

MEADOR, R. Cogeneration and district heating: an energy-efficiency partnership. Ann Arbor Sci: Ann Arbor, 1981.

MINISTERIO DE VIVIENDA Y URBANISMO (MINVU). Plan de Reconstrucción. Disponible en Internet: http://minvu.cl/opensite_20101001180448.aspx

PEREZ, R.; INEICHEN, P.; MOORE, K.; KMIECIKM, C. \& GEORGE, R. A new operational for satellite-derived irradiances: description and validation. Solar Energy, 2002, № 73, p. 307-317.

REDWEICK, P.; CATITA, C. \& BRITO, M. Solar energy potential on roofs and facades in an urban landscape. Solar Energy, 2013, N ${ }^{\circ}$ 97, p. 332-341.

REINHART, C.; BERLIN, E.; JAKUBIEC, A. \& WAISSBLUTH, N. The Mapdwell Project. Disponible en Internet: http://es.mapdwell.com/

SALINAS, E. y PÉREZ, L. Procesos urbanos recientes en el Área Metropolitana de Concepción: transformaciones morfológicas y tipologías de ocupación. Revista de Geografía Norte Grande, 2011, № 49, p. 79-97.

DAS SOLARKATASTER BREMEN (SB). Disponible en Internet: http://solarkatasterbremen.de

SUSTAINABLE ENGINEERING LAB (SEL) AT THE EARTH INSTITUTE, COLUMBIA UNIVERSITY. Building Energy Consumption for NYC. Disponible en internet: http://modi. mech.columbia.edu/resources/nycenergy/

SUN, Y.; HOF, A.; WANG, R.; LIU, J.; LIN, Y. \& YANG, D. GIS-based approach for potential analysis of solar PV generation at the regional scale: A case study of Fujian Province. Energy Policy, 2013, № 58, p. 248-259. 
MAPA ENERGÉTICO-SOLAR DE CONCEPCIÓN: CARTOGRAFÍA URBANA DEL CONSUMO ENERGÉTICO Y CAPTACIÓN SOLAR EN EDIFICACIONES RESIDENCIALES DE CONCEPCIÓN, CHILE

UNIVERSIDAD POLITÉCNICA DE MADRID (UPM) \& POLIS. Methodology for Identification of Detailed Solar Potential of Urban Areas, 2010. Disponible en Internet: http://www.polis-solar.eu/
WIDÉN, J., WÄCKELGARD, E., PAATERO, J. \& LUND, P. Impacts of different data averaging times on statistical analysis of distributed domestic photovoltaic systems. Solar Energy, 2010, No 84, p. 492-500. 
